\title{
Krigagem ordinária para predição de vazões em locais sem dados: proposta de nova
}

\section{metodologia}

\author{
Ordinary krigage for flow prediction in locations without data: proposal for a new methodology \\ Krigage ordinario para la predicción de flujos en ubicaciones sin datos: propuesta de una nueva \\ metodología
}

Recebido: 28/12/2021 | Revisado: 03/01/2022 | Aceito: 03/02/2022 | Publicado: 05/02/2022

Camila Machado

ORCID: https://orcid.org/0000-0001-6382-7636 Universidade Federal de Mato Grosso do Sul, Brasil E-mail: eng.cdourado@gmail.com

Fábio Veríssimo Gonçalves

ORCID: https://orcid.org/0000-0003-2665-7687 Universidade Federal de Mato Grosso do Sul, Brasil E-mail: fabio.goncalves@ufms.br

Alisson Ribeiro

ORCID: https://orcid.org/0000-0002-0478-977X Universidade Federal de Mato Grosso do Sul, Brasil E-mail: geotec.ribeiro@gmail.com

Fabrício Bau Dalmas

ORCID: https://orcid.org/0000-0001-7547-6642 Universidade Guarulhos, Brasil E-mail: fbdalmas@gmail.com

Antônio Conceição Paranhos Filho

ORCID: https://orcid.org/0000-0002-9838-5337

Universidade Federal de Mato Grosso do Sul, Brasil E-mail: antonio.paranhos@pq.cnpq.br

\begin{abstract}
Resumo
O conhecimento da disponibilidade hídrica dos corpos hídricos é de grande importância para sua gestão e planejamento das atividades que dependem destes. Entretanto, grandes dificuldades são encontradas no decorrer deste processo, visto a ausência de dados na grande maioria das bacias hidrográficas. Nesse trabalho é apresentada uma técnica que tem se demonstrado eficiente nesse sentido e tem sido aplicada pelo órgão ambiental de Mato Grosso do Sul, porém que pode também ser aplicada em outras bacias hidrográficas de outros Estados: o mapeamento de vazões mínimas que, além de solucionar este problema da ausência de dados, confere agilidade ao procedimento de obtenção de dados hidrológicos. Esta pesquisa tem objetivo de apresentar uma metodologia para elaboração de mapeamento de dispersão em curvas de vazões mínimas através da krigagem ordinária, auxiliando assim o processo de estimativa de vazões, uma vez que serve como ferramenta para coleta e geração de informações de vazões de referências através da simples inserção da coordenada geográfica do ponto em interesse. Foram utilizados dados de 79 estações fluviométricas e gerados os mapas para o Estado de Mato Grosso do Sul. Os resultados obtidos foram considerados satisfatórios com base na validação cruzada, onde foram obtidos coeficientes de dispersão em torno de 0,70 .
\end{abstract}

Palavras-chave: Disponibilidade hídrica; Regionalização de vazões; Vazões mínimas.

\begin{abstract}
The knowledge of water availability in water bodies is of great importance for their management and planning of activities that depend on them. However, great difficulties are encountered during this process, given the lack of data in the vast majority of watersheds. This work presents a technique that has been shown to be efficient in this regard and has been applied by the environmental agency of Mato Grosso do Sul State, but which can also be applied in watersheds of other states: the mapping of minimum flows that, in addition to solving this problem of absence of data, makes the procedure for obtaining hydrological data agile. This research aims to present a methodology for preparing dispersion mapping in minimum flow curves through ordinary kriging, thus helping the flow estimation process, as it serves as a tool for collecting and generating information on reference flow rates by simply inserting the geographic coordinate of the point of interest. Data from 79 fluviometric stations were used and maps were generated for the State of Mato Grosso do Sul. The results obtained were considered satisfactory based on cross validation, where dispersion coefficients around 0.70 were obtained.
\end{abstract}

Keywords: Water availability; Ordinary kriging; Minimum flows. 


\section{Resumen}

El conocimiento de la disponibilidad de agua en los cuerpos de agua es de gran importancia para su gestión y planificación de las actividades que dependen de ellos. Sin embargo, se encuentran grandes dificultades durante este proceso, dada la falta de datos en la gran mayoría de las cuencas hidrográficas. Este trabajo presenta una técnica que ha demostrado ser eficiente en este sentido y ha sido aplicada por la agencia ambiental de Mato Grosso do Sul, pero que también puede ser aplicada en otras cuencas hidrográficas de otros estados: el mapeo de caudales mínimos que, además de solucionar este problema de falta de datos flexibiliza el procedimiento para la obtención de datos hidrológicos. Esta investigación tiene como objetivo presentar una metodología para la elaboración de mapas de dispersión en curvas de flujo mínimo mediante kriging ordinario, ayudando así al proceso de estimación de flujo, ya que sirve como herramienta para recolectar y generar información de flujo de referencia a través de la simple inserción de la coordenada geográfica del punto. de interés. Se utilizaron datos de 79 estaciones fluviométricas y se generaron mapas para el estado de Mato Grosso do Sul. Los resultados obtenidos se consideraron satisfactorios en base a la validación cruzada, donde se obtuvieron coeficientes de dispersión alrededor de 0,70 .

Palabras clave: Disponibilidad de agua; Regionalización de flujos; Caudales mínimos.

\section{Introdução}

Em decorrência da preocupação com a escassez dos recursos hídricos e a necessidade de garantir a disponibilidade de água as presentes e futuras gerações, em 1997 foi instituída a Política Nacional de Recursos Hídricos, Lei nº 9433/1997 (Brasil, 1997), mudando a perspectiva de gestão deste bem público.

Na gestão de recursos hídricos, um aspecto de extrema importância é o conhecimento da disponibilidade hídrica, pois é através dela que é possível se fazer a análise do balanço hídrico e distribuir de forma adequada aos usuários a quantidade de água necessária para seus empreendimentos. A análise da disponibilidade hídrica é realizada por meio de estimativas de vazões utilizando-se de métodos estatísticos, de forma a procurar representar de forma mais coerente possível a quantidade de água disponível no curso d'água.

Mais especificamente, a estimativa de vazões de estiagem é feita pela inferência estatística das séries históricas de estações de monitoramento mantidas pela União e pelos Estados. Entretanto devido ao elevado custo destas estações, e a extensão do território nacional, estas estão instaladas em grandes áreas de drenagem, o que dificulta a estimativa de vazões nas pequenas bacias hidrográficas.

Para resolver este problema de falta de dados em pequenas bacias, são utilizadas diversas metodologias de regionalização de dados hidrológicos, processo este que consiste na transferência de informações hidrológicas de um local para outro, desde que os pontos estejam dentro de uma área de semelhança hidrológica (Tucci, 2002).

Seguindo o assunto supracitado, o presente estudo tem o objetivo a apresentação de uma metodologia de confecção de mapa de dispersão em curvas de vazões mínimas, tendo como estudo de caso o Estado de Mato Grosso do Sul, através aplicação da metodologia da krigagem ordinária.

\subsection{Regionalização de dados de vazão de Bacias hidrográficas}

Diversas metodologias de regionalização têm sido estudadas e são encontradas facilmente na literatura técnica (Maciel et al., 2019; Andrade et al., 2020; Silva \& Manzione, 2020). Tais estudos apresentam métodos baseados em aplicações estatísticas, determinação de equações para vazões através de regressão, que relacionam os fatores que influenciam na distribuição destas, sendo estes fatores, variáveis explicativas, simulações hidrológicas e através de interpolação em sistemas de informação geográficas.

Contudo, os métodos anteriormente citados ainda não conferem agilidade aos processos de estimativa de vazões, devido à necessidade da semelhança hidrológica entre as regiões para a aplicação das metodologias tradicionais. Estudos mais recentes apontam as técnicas geoestatísticas como um eficiente método para predição de valores de vazões em locais sem dados, uma vez 
que a integração destas técnicas de interpolação às tecnologias de Sistemas de Informação Geográfica (SIG) tornou mais eficiente a elaboração de mapas através da análise e interpolação de dados espacialmente distribuídos.

A krigagem, processo geoestatístico muito utilizado para predizer valores em locais sem dados, vem sendo utilizado tanto para estimação de vazões características máximas e mínimas, quanto para predição de séries históricas completas (Farmer, 2016). A principal vantagem da utilização desta técnica é a de que não há necessidade da obtenção das demais variáveis explicativas geralmente utilizadas em outros modelos matemáticos, que são de difícil obtenção e por vezes apresentam baixa correlação. Além disso, nesse processo não há necessidade de delimitação de regiões hidrologicamente homogêneas, trabalho que requer tempo e conhecimento de grande quantidade de dados hidrológicos. Como resultado desta interpolação, pode ser obtido, um mapa de isovalores e este sistema de mapeamento de vazões mínimas garante maior facilidade para obtenção e aplicação destes dados hidrológicos.

\section{Metodologia}

Esta é uma pesquisa experimental e a metodologia utilizada na elaboração desta pesquisa foi a quali-quantitativa (Pereira et. al, 2018), na qual foram realizadas coletas de dados secundários, oriundos de órgãos oficiais, tais como IBGE e IMASUL, que foram apresentadas na forma de mapas temáticos. A krigagem foi utilizada como técnica estatística dos dados secundários coletados.

\section{1 Área de Estudo e Cálculo das Vazões Mínimas de Referência}

A área de estudo onde o modelo foi aplicado é o Estado de Mato Grosso do Sul, que possui extensão de 357.145,534 km² (IBGE, 2011). Foram selecionados dados de vazão das estações hidrometeorológicas que estão distribuídas pelo território estadual e são mantidas pela Agência Nacional de Águas (ANA) em parceria com o Instituto de Meio Ambiente de Mato Grosso do Sul (IMASUL). De acordo com a Resolução No 32 do Conselho Nacional de Recursos Hídricos (CNRH) de 2003 (Brasil, 2003), o Estado contém em seu território duas das doze Regiões Hidrográficas do Brasil, sendo estas a Região Hidrográfica do Paraguai, ou Bacia do Rio Paraguai e a Região Hidrográfica do Paraná, ou Bacia do Rio Paraná.

As duas Bacias citadas possuem diferenças significativas em seus escoamentos, sendo que a Bacia do Rio Paraguai possui menor escoamento devido à baixa capacidade de drenagem do Pantanal, que recebe as vazões do Planalto e retém grande parte do volume de água, diminuindo as vazões que passam para jusante, caracterizando a variabilidade sazonal significativa (Mato Grosso do Sul, 2010). Outros fatores que influenciam também nas diferenças de escoamento das bacias e na sazonalidade de vazões disponíveis nos cursos hídricos, são os aspectos climatológicos, as precipitações e a evapotranspiração.

Para o cálculo das vazões mínimas de referência, foram utilizados dados de estações fluviométricas disponíveis no inventário da Agência Nacional de Águas (ANA, 2016) acessado através do portal Hidroweb (http://hidroweb.ana.gov.br). Os dados das séries históricas das estações passaram por uma triagem inicial, garantindo que fossem aproveitadas apenas estações com mais de cinco anos de dados consistidos, mínimo necessário para um estudo hidrológico (Tucci, 2002), sendo assim um levantamento inicial apontou um total de 79 estações nessa situação, que podem ser observadas na Figura 1. As áreas de drenagem das estações variam de 315 a $804.000 \mathrm{~km}^{2}$. 
Figura 1. Área de Estudo (Elaborado em SIRGAS 2000 - UTM 21S).

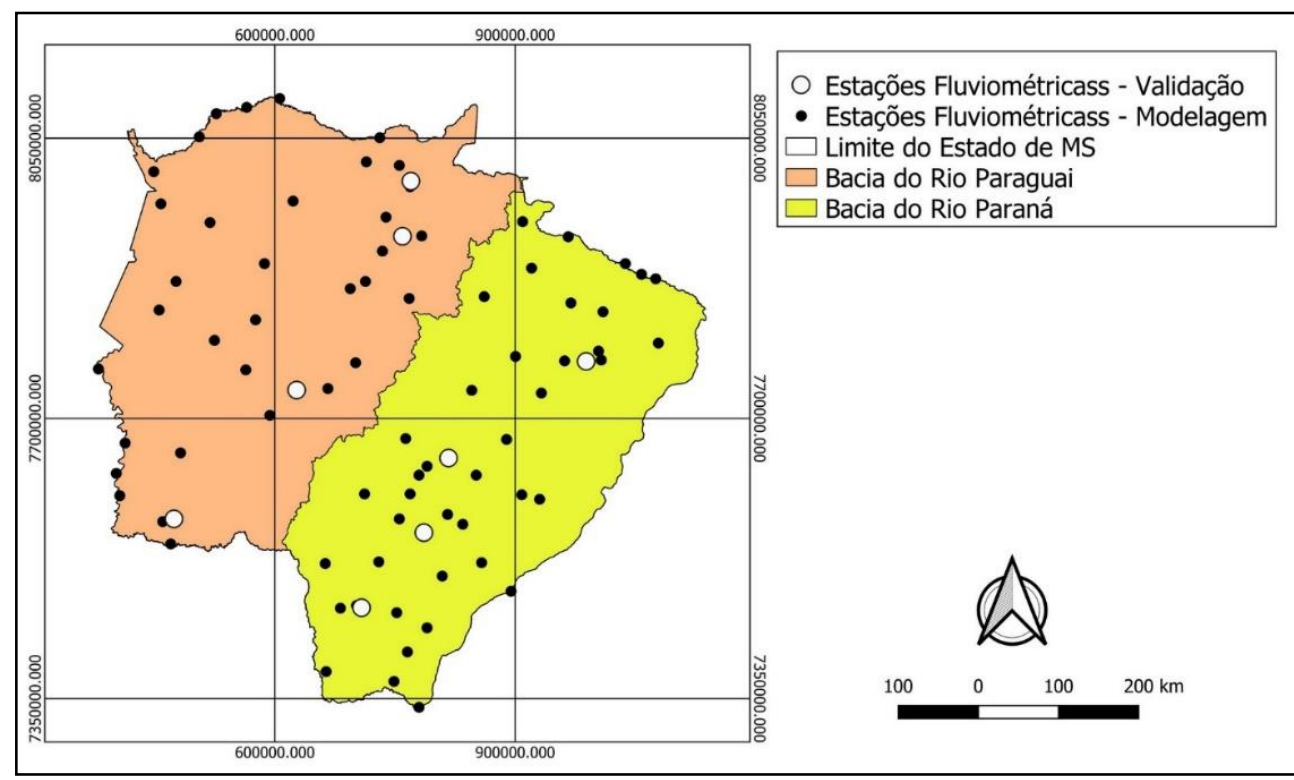

Fonte: Autores.

Para todas as estações foram calculadas as vazões com permanência de $95 \%$ do tempo, vazão de referência para o Estado, conforme a Resolução CERH-MS 025/2014 (Mato Grosso do Sul, 2014). A primeira etapa da construção das curvas de permanência ocorreu através da definição do intervalo de tempo da variável e do período de dados que a curva representa. Para cada estação fluviométrica foram utilizados os dados diários de vazões e a totalidade de anos observados. As séries amostradas têm períodos de dados variando de 1910 até 2007.

Os anos com falhas ou sem valores observados foram descartados para não haver a necessidade de realizar o preenchimento de falhas. A opção pelo não preenchimento de falhas se deu em conformidade com as recomendações de Tucci (2002), uma vez que os preenchimentos através de técnicas de regressão podem introduzir incertezas tendenciosas, causando assim um enviesamento do resultado.

Para a elaboração do mapa de vazões, os valores obtidos anteriormente foram transformados em rendimentos específicos, através da divisão do valor da Q95 pela área de drenagem (Ad) do referido posto fluviométrico. Esta conversão garantiu ao trabalho valores homogêneos, que caracterizam toda a região da bacia hidrográfica em que o posto se encontra, e assim, amenizou as diferenças elevadas de valores da Q95 em função das amplas diferenças entre as áreas de drenagens dos postos utilizados. Sem a conversão a variação amostral seria maior, acarretando maiores imprecisões no decorrer dos cálculos de interpolação.

\subsection{Krigagem Ordinária e Modelagem Computacional}

O uso da krigagem para estimativa de vazões em locais sem dados partiu da premissa de que vazões são variáveis regionalizadas, ou seja, os valores amostrados apresentam correlação entre si em decorrência da correlação espacial. As variáveis regionalizadas possuem quatro características a mais que as distinguem de variáveis aleatórias, que são a localização geográfica, o suporte, a continuidade e a presença ou não de anisotropia.

A localização das vazões específicas está expressa pelo georreferenciamento dos dados, o suporte é sempre o mesmo, pois se trata de um dado pontual. A premissa da continuidade e dependência espacial está baseada na hipótese inicial adotada, que presume que os dados variam de forma contínua, ou seja, quanto maior a distância entre dois pontos amostrais, menor é a influência entre eles, até uma determinada distância onde não ocorre mais esta influência. Para cada vizinho do ponto em 
interesse, são atribuídos pesos de influência no resultado "predito" de acordo com a distância entre o ponto amostrado e o local (Yamamoto, 2020).

A anisotropia foi estudada no decorrer da modelagem do semivariograma experimental e consiste na diferença do comportamento da variação entre os dados, de acordo com a direção estudada. A vantagem da utilização da krigagem como estimador está justamente na capacidade de reconhecimento desta anisotropia e a utilização dela nos cálculos de estimativa. A estatística passa a ser aplicada a dados georreferenciados e a predição dos dados de vazões específicas em locais sem informação serão baseados nas informações vizinhas.

Definições da direção e do raio de busca, ou seja, qual o limite que um ponto interfere no outro, dependem das características do domínio estudado, ou seja, estudou-se a variância espacial dos dados amostrados para saber como ocorre a variação entre esses dados. Partindo do pressuposto apresentado, a metodologia da krigagem se resume na Equação Fundamental, ou Função Semivariograma, em relação expressa na Eq. 1 (Yamamoto, 2020):

$$
\text { Equação 1. } 2 \gamma_{(n)}=\frac{1}{n} \sum_{i=1}^{n} \quad\left(Z_{x i}-Z_{(x i+h)}\right)^{2}
$$

onde:

$2 \gamma_{(n)}=$ Variância.

$\mathrm{Z}=$ valor amostral.

$\mathrm{h}=$ distância entre o ponto e o vizinho.

A modelagem começou pelo estudo do semivariograma experimental, onde inicialmente realiza-se o estudo de várias direções em busca do par de direções ortogonais, onde se encontra a maior anisotropia. As características modeladas no estudo do semivariograma experimental são o efeito pepita, a amplitude e o patamar. O efeito pepita é um conceito teórico do valor da variância espacial quando a distância entre os vizinhos tende a zero. A amplitude é caracterizada pela distância máxima onde as amostras possuem dependência espacial entre si e o patamar é o valor onde a variância espacial se estabiliza. A diferença entre os valores das amplitudes e dos patamares em decorrência das diferentes direções analisadas é que caracteriza os tipos de anisotropia (Yamamoto, 2020).

Uma vez estudado o semivariograma experimental, é possível escolher o teórico que será utilizado no processo da krigagem. Ressalta-se aqui que o modelo escolhido é fruto do semivariograma experimental, que por sua vez resulta dos dados amostrados. Antes da aplicação da krigagem, é realizada a validação cruzada, cujo objetivo principal é aferir a qualidade do modelo escolhido, definindo assim os parâmetros de vizinhança que serão utilizados para garantir a representatividade espacial. É considerada uma pré-krigagem, na qual um a um os dados de amostra são retirados e estimados pelo modelo escolhido e, assim, faz-se a aferição da fidelidade do modelo ao resultado original. Por fim, é aplicado o estimador da krigagem, definido pela Eq. 2 (Yamamoto, 2020):

$$
\text { Equação 2. } Z_{\left(x_{0}\right)}^{*}=\sum_{i=1}^{n} \quad \delta_{i} Z_{x i}
$$

onde:

$Z^{*}=$ valor estimado

$\delta_{i}=$ ponderador (variância)

$Z=$ valor da amostra

Optou-se pela utilização da krigagem ordinária, pois neste processo assume-se médias móveis, fazendo com que haja minimização da média do erro. Todo o processo de cálculo foi desenvolvido através do software Geovisual (Yamamoto, 2015). Para realização das modelagens e posterior obtenção do mapa de vazões, o estudo foi dividido em duas etapas. Com as 79 amostras iniciais, sendo destas 36 pertencentes à Bacia do Paraguai e 43 à Bacia do Rio Paraná, foi assim gerado o mapa de vazões para o Estado de Mato Grosso do Sul. 
Posteriormente os cálculos foram realizados separadamente de acordo com as Regiões Hidrográficas existentes no Estado. Nesta segregação do espaço amostral, pontos que possuíam valores muito discrepantes da maioria do novo espaço amostral foram descartados evitando correlações espúrias. Foram realizadas duas simulações, em áreas de tamanhos diferentes, sendo que para a confecção do mapa da Bacia do Paraguai foram utilizadas 33 estações; e para a Bacia do Rio Paraná foram consideradas 42 estações.

Inicialmente, para os dois casos simulados foram construídos os semivariogramas para quatro direções principais, de $0^{\circ}, 45^{\circ}, 90^{\circ}$ e $135^{\circ}$, utilizando como tolerância angular de $22,5^{\circ}$ para a realização da busca de pares de pontos. Foi verificado que para todos os casos, o par de direções ortogonais que possuía maior anisotropia foi para as direções de $0^{\circ}$ e $90^{\circ}$. Assim, foi recalculado o semivariograma experimental considerando as duas direções citadas acima e uma tolerância angular de $45^{\circ}$.

Para os dados da Bacia do Rio Paraguai foi verificada a presença de anisotropia mista. Já para os dados da Bacia do Rio Paraná foi verificada a presença de anisotropia zonal. O próximo passo foi a construção do semivariograma teórico, através da correção da anisotropia pela adoção de duas estruturas para encontrar a estrutura resultante. A Tabela 1 apresenta os parâmetros de ajuste de modelo do semivariograma teórico.

Tabela 1. Parâmetros do Semivariogramas Teóricos dos casos de estudo em Mato Grosso do Sul.

\begin{tabular}{lcccccc}
\hline & \multicolumn{2}{c}{ Estado de MS } & \multicolumn{2}{c}{ Bacia do Rio Paraguai } & \multicolumn{2}{c}{ Bacia do Rio Paraná } \\
\hline Estrutura & 1 & 2 & 1 & 2 & 1 & 2 \\
Azimute & $90^{\circ}$ & $90^{\circ}$ & $90^{\circ}$ & $90^{\circ}$ & $0^{\circ}$ & $0^{\circ}$ \\
Modelo & Esférico & Esférico & Esférico & Esférico & Gaussiano & Exponencial \\
Variância Espacial & 9,86 & 14,14 & 2,07 & 1,26 & 3,99 & 1,95 \\
Amplitude Máxima & 1,19 & $1,00 \mathrm{E}+30$ & 1,62 & $1,00 \mathrm{E}+30$ & 0,53 & $1,00 \mathrm{E}+30$ \\
Amplitude Mínima & 4,24 & 4,24 & 1,42 & 1,42 & 0,53 & 0,53 \\
\hline
\end{tabular}

Fonte: Autores.

Uma vez realizado o ajuste do modelo teórico do semivariograma realizou-se a validação cruzada e obteve-se o coeficiente de regressão estimado. Ao final foi executado o algoritmo da krigagem ordinária, para obtenção dos mapas de rendimentos específicos desejados. Os resultados obtidos foram então tratados no SIG Qgis (QGIS Development Team, 2015) para melhor visualização e convertidos então em shapefiles. Além dos mapas de valores estimados, foi possível obter o mapa das incertezas associadas, sendo estas o desvio padrão de krigagem e o desvio padrão de interpolação. Estes valores são úteis para estimar a confiabilidade do valor estimado e a incerteza associada a este valor, servindo como balizador para a tomada de decisão.

\section{Resultados e Discussão}

Após análise da consistência dos dados obtidos através do portal Hidroweb, foram calculadas as vazões de permanência (Q95) e rendimento específico (Q95) para as 79 estações utilizadas na modelagem. Os valores obtidos para os rendimentos específicos nos postos pertencentes à Bacia do Rio Paraguai são mais baixos que aqueles pertencentes à Bacia do Rio Paraná. Tal fato reafirma o comportamento hidrológico diferente nestas duas regiões hidrográficas. Estes valores mais baixos decorrem da presença da Região do Pantanal e sua baixa capacidade de drenagem, aliadas ao fato do relevo desta região ser marcado pela presença das menores altitudes e das baixas declividades de seus corpos hídricos. Outros fatores não menos importantes são a evapotranspiração, que comparada à Bacia do Rio Paraná, ocorre de forma mais acentuada nesta região hidrográfica, além da presença dos menores índices de precipitação e sua distribuição heterogênea. 
Esta diferença entre as duas regiões é um fator que explica a complexidade da gestão 276 de recursos hídricos no estado do Mato Grosso do Sul, uma vez que temos regiões com 277 comportamento hidrológico e disponibilidade de água para uso distintos, havendo assim a 278 necessidade da flexibilização dos critérios de uso, e maior cuidado no estudo da transferência 279 de informações de dados hidrológicos de uma região para a outra e elaboração de modelos 280 hidrológicos.

Analisando a estatística básica dos dados, apresentada na tabela 2, é possível observar 288 que a Bacia do Rio Paraná possui menor variabilidade dos dados, indicando maior 289 homogeneidade. Essa homogeneidade acarreta uma precisão maior na estimativa, esperando-290 se assim resultados com menores erros nesta região.

Tabela 2. Estatística Básica efetuada sobre os dados de vazão de Mato Grosso do Sul.

\begin{tabular}{|c|c|c|c|}
\hline & Estado MS & Bacia do Rio Paraguai & Bacia do Rio Paraná \\
\hline Mínimo (L/s.km²) & 0,14 & 0,14 & 3,87 \\
\hline Máximo (L/s.km²) & 19,05 & 6,63 & 14,12 \\
\hline Média (L/s.km²) & 6,61 & 2,83 & 8,87 \\
\hline Mediana (L/s.km²) & 5,66 & 2,68 & 8,84 \\
\hline Variância (L/s.km²) & 17,93 & 3,05 & 8,69 \\
\hline Desvio Padrão (L/s.km²) & 4,24 & 1,74 & 2,95 \\
\hline Coeficiente de Variação & 0,64 & 0,62 & 0,33 \\
\hline Assimetria & 0,47 & 0,42 & 0,15 \\
\hline
\end{tabular}

Fonte: Autores.

\subsection{Krigagem Ordinária}

A malha formada pelos pontos amostrais não foi regular, o que favoreceu o aparecimento de imprecisões ao longo da modelagem. Também ocorreu a presença de grandes vazios amostrais, comprometendo desta forma a vizinhança desses locais, uma vez que os pontos que ali se encontram, possuem menos vizinhos para ser considerados no decorrer dos cálculos. Esse vazio entre os dois conjuntos de dados é o divisor de bacias, região de nascentes, portanto, sem estações de medição.

Essa irregularidade da malha é fruto de um não planejamento da amostragem, decorrência da necessidade de se utilizar dados dos postos fluviométricos disponíveis e estes refletem a baixa densidade de informações de vazões ao longo do território estadual. Foi realizada a regularização da malha no decorrer do processo de procura de anisotropias e modelagem dos semivariogramas, encontrando assim um modelo que representasse de modo eficaz a variabilidade dos dados. A Figura 2 apresenta os resultados dos semi-semivariogramas, experimental e teórico para os casos de estudo. No eixo horizontal temos representadas as distâncias entre as amostras e no eixo vertical a variação entre os valores de cada amostra. As linhas irregulares apresentam os modelos experimentais para as direções que apresentaram maior anisotropia, e as linhas contínuas apresentam o modelo teórico. Os pontos de inflexão dos modelos teóricos representam os parâmetros utilizados como ponderador. 
Figura 2. Semivariogramas para os casos de estudo.

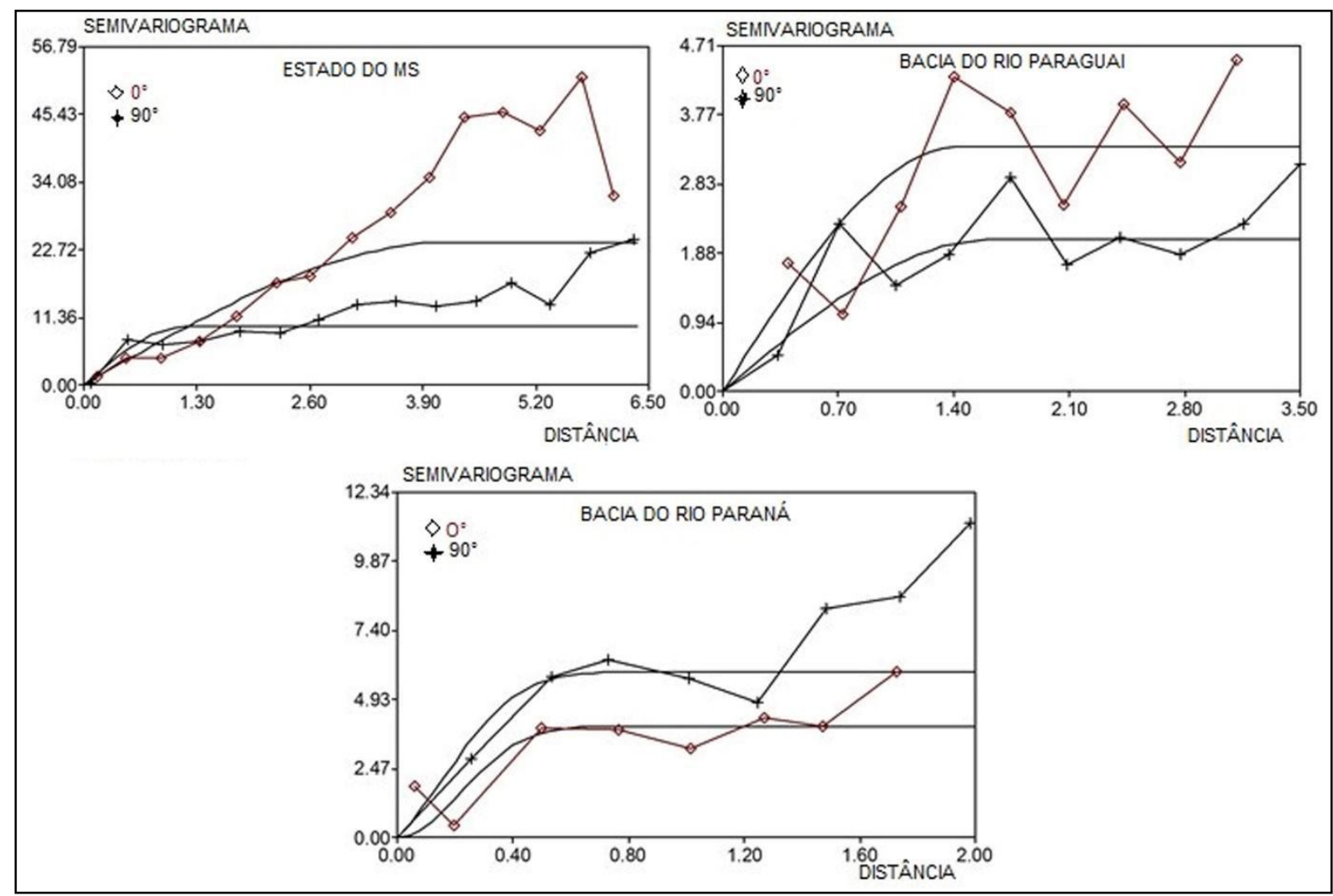

Fonte: Autores.

O melhor ajuste para o semivariograma no da Bacia do Rio Paraguai foi o modelo esférico, assim como nas metodologias desenvolvidas por Farmer (2016) e Castiglioni et al. (2011), fato explicado pela variação inicial linear no semivariograma. Já para o caso da Bacia do Rio Paraná o melhor ajuste foi a mistura dos modelos gaussianos e exponencial, devido ao comportamento parabólico dos dados próximos à origem do semivariograma.

A forma de atestar a validade dos modelos obtidos foi através da validação cruzada, procedimento no qual cada ponto amostral foi retirado inicialmente e assim amostrado pelo modelo e posteriormente foi realizada a comparação entre os valores originais e os valores obtidos pela krigagem.

Na Figura 3 são apresentados os resultados dos valores de dispersão obtidos pela validação. As melhores correlações obtidas foram para o modelo que abrange todo o Estado de MS e o modelo para a Bacia do Rio Paraná, esperando-se assim que estes casos apresentem menores erros de estimação que o modelo para a Bacia do Rio Paraguai. Entretanto, os três valores obtidos, em torno de 0,70 , são aceitáveis levando em consideração a variabilidade dos dados e fornecem resultados confiáveis. 
Figura 3. Resultado da Validação Cruzada para os casos de estudo.

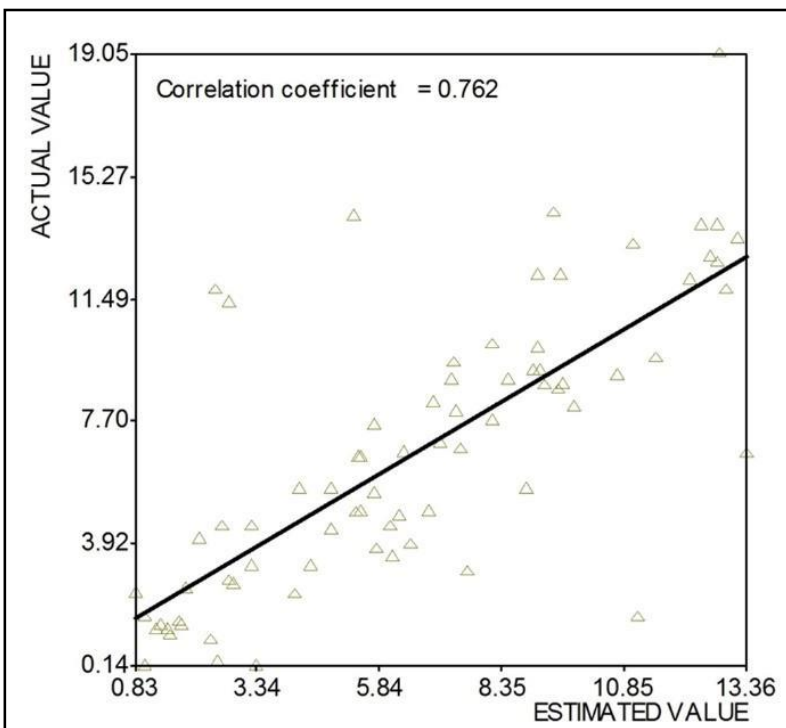

Estado do MS

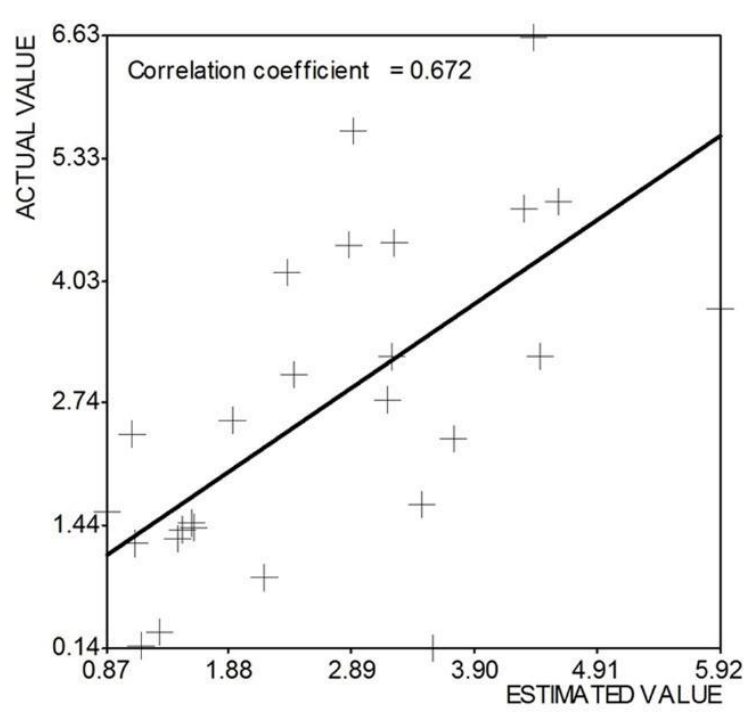

Bacia do Rio Paraguai

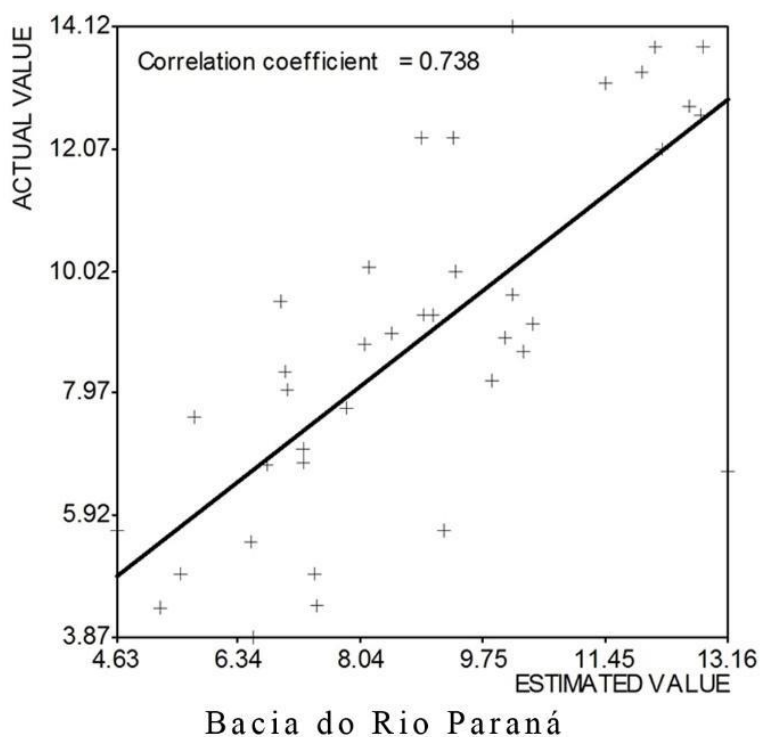

Fonte: Autores.

As melhores correlações obtidas foram para o modelo que abrange todo o Estado de 340 MS e o modelo para a Bacia do Rio Paraná, esperando-se assim que estes casos apresentem 341 menores erros de estimação que o modelo para a Bacia do Rio Paraguai. Entretanto, os três 342 valores obtidos, em torno de 0,70, são aceitáveis levando em consideração a variabilidade dos 343 dados.

As Figuras 4, 5 e 6 apresentam os mapas de valores de rendimento específico estimado (Q95*) para os três casos estudados, sendo o caso 1 a modelagem para o Estado de MS; o caso 2 a modelagem para Bacia do Rio Paraguai; e o caso 3 a modelagem para Bacia do Rio Paraná. 
Figura 4. Caso 1 Resultado de Valores de Rendimento Específico Estimado (Q95*) para o Estado de MS (Elaborado em SIRGAS 2000 - UTM 21S).

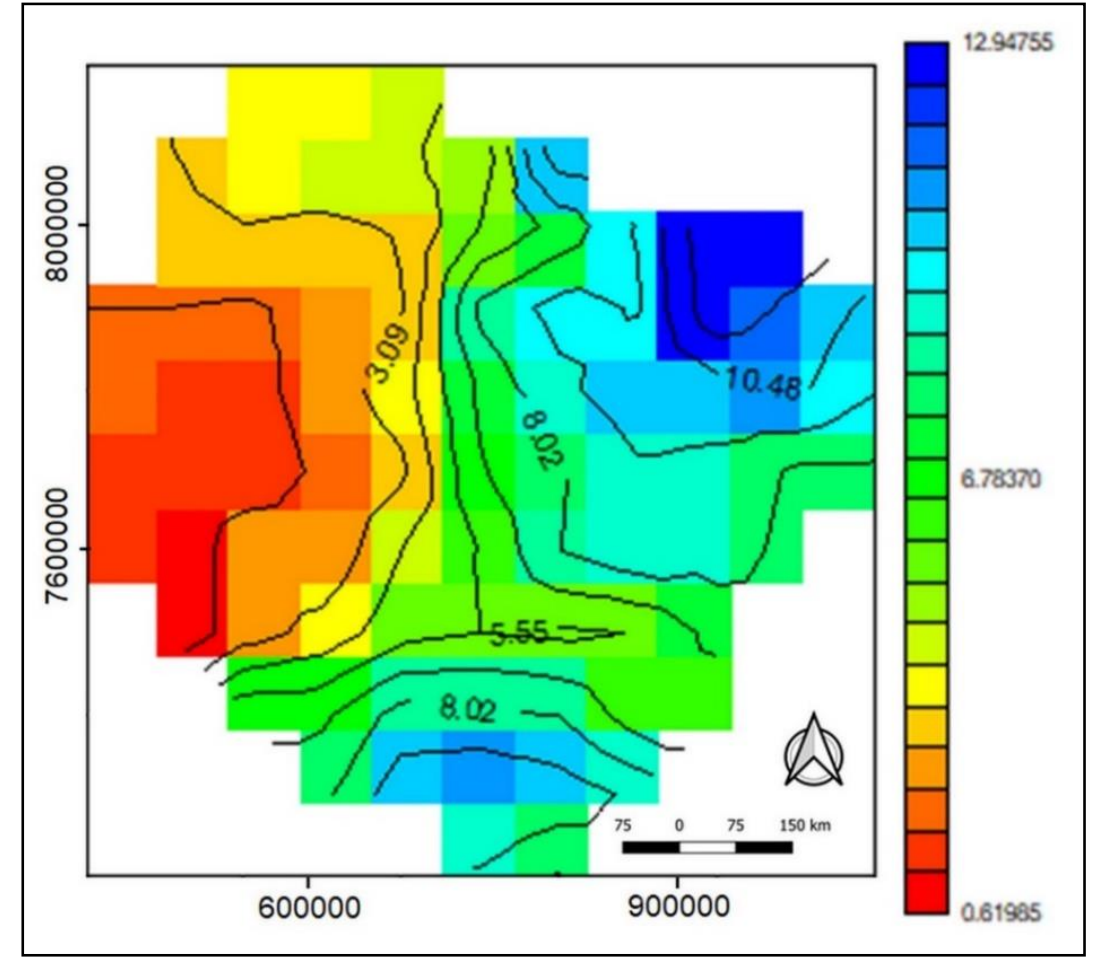

Fonte: Autores.

Figura 5. Caso 2 Resultado de Valores de Rendimento Específico Estimado (Q95*) para a Bacia do Rio Paraguai (Elaborado em SIRGAS 2000 - UTM 21S).

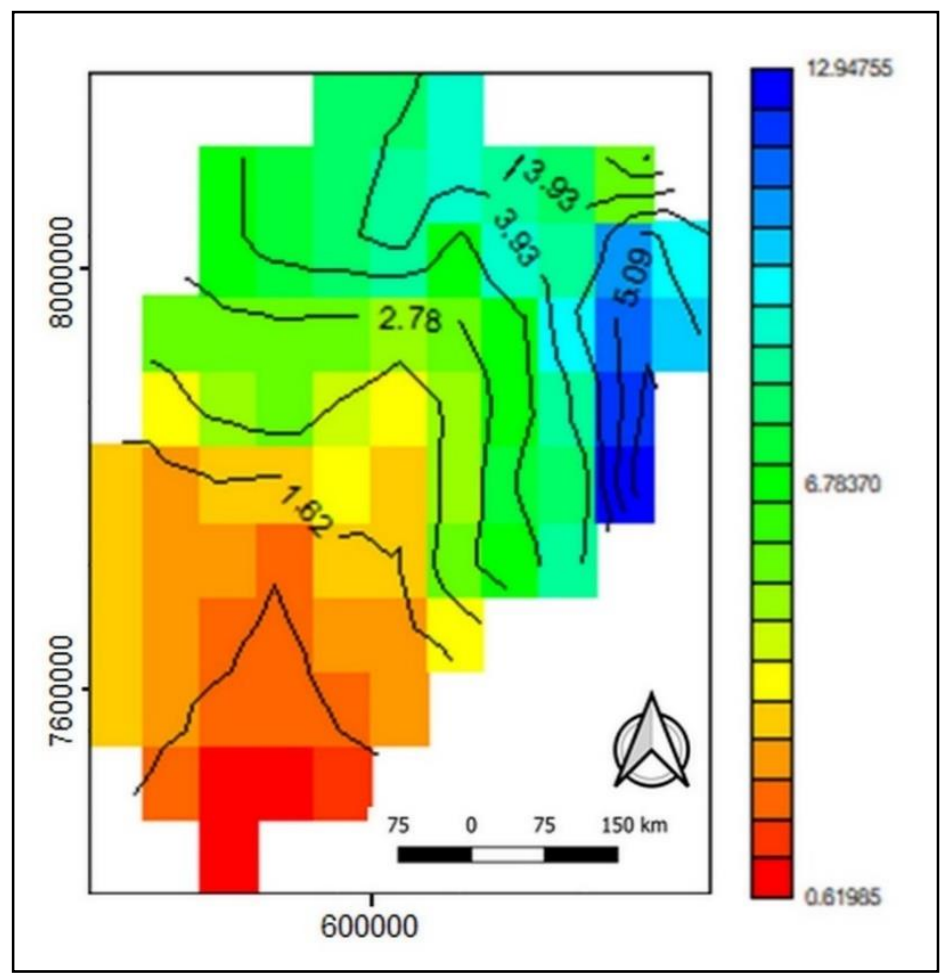

Fonte: Autores. 
Figura 6. Caso 3 Resultado de Valores de Rendimento Específico Estimado (Q95*) para a Bacia do Rio Paraná (Elaborado em SIRGAS 2000 - UTM 21S).

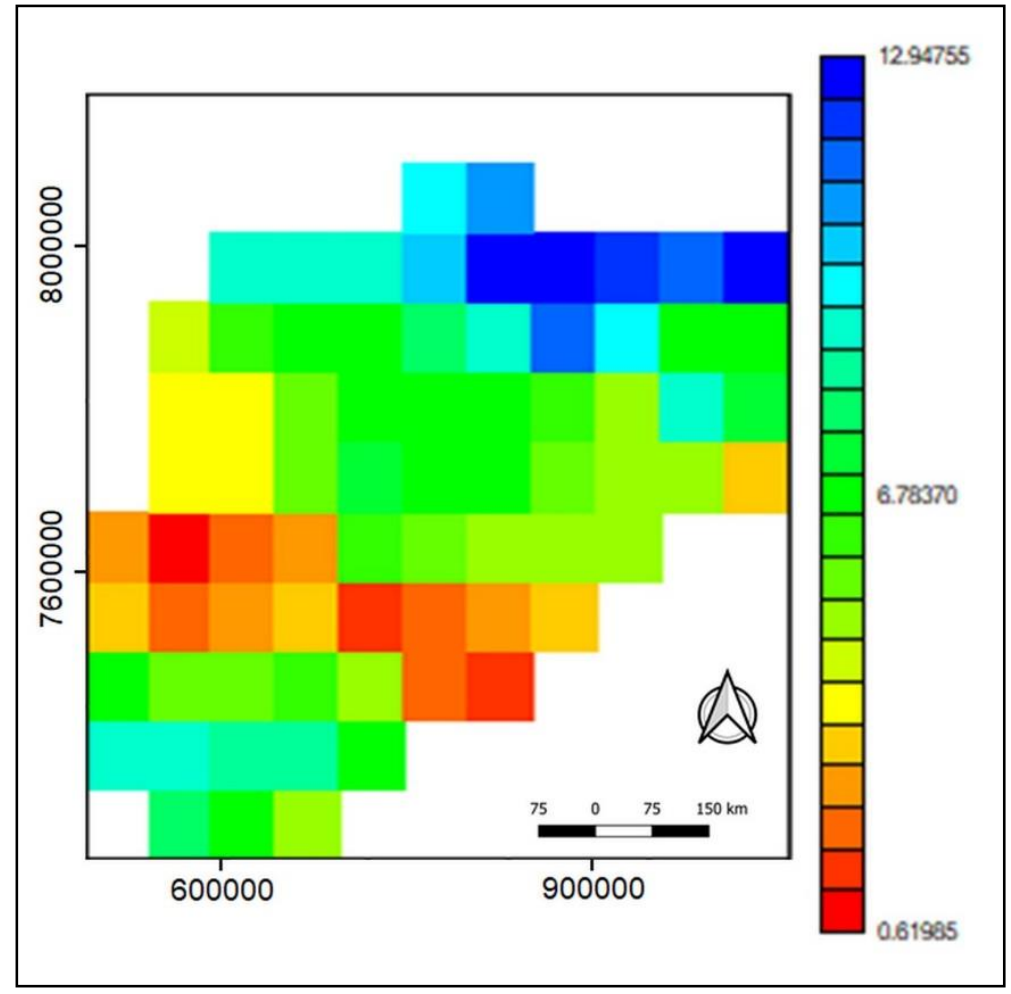

Fonte: Autores.

Foram obtidos também resultados para os desvios de interpolação e desvio de krigagem. Os resultados extraídos para as estações que já possuíam valores observados para posterior comparação são apresentados nas Tabelas 3 (Caso 1), 4 (Caso 2) e 5 (Caso 3).

Tabela 3. Caso 1 - Resultados de Krigagem Ordinária para o Estado de MS.

\begin{tabular}{cccccc}
\hline PONTO & Estações ID ANA & $\begin{array}{c}\text { Q95 } \\
\left(\mathbf{L} / \mathbf{s . k m} \mathbf{k m}^{2}\right)\end{array}$ & $\begin{array}{c}\mathbf{Q 9 5}^{*} \\
\left(\mathbf{L} / \mathbf{s . k m}^{2}\right)\end{array}$ & $\begin{array}{c}\text { Desvio de } \\
\text { Interpolação }\end{array}$ & $\begin{array}{c}\text { Desvio de } \\
\text { Krigagem }\end{array}$ \\
\hline 1 & 66855000 & 7,73 & 5,64 & 1,27 & 2,21 \\
2 & 66945000 & 2,11 & 1,58 & 0,37 & 2,12 \\
3 & 66840000 & 7,24 & 10,09 & 3,74 & 2,62 \\
4 & 67175000 & 0,27 & 0,74 & 1,93 & 2,14 \\
5 & 64703000 & 9,23 & 10,28 & 1,19 & 2,06 \\
6 & 63950250 & 10,41 & 8,51 & 0,3 & 2,08 \\
7 & 64610000 & 7,48 & 8,89 & 1,06 & 2,18 \\
8 & 63390000 & 7,39 & 12,55 & 1,58 & 2,09 \\
\hline
\end{tabular}

Fonte: Autores. 
Research, Society and Development, v. 11, n. 2, e54511225185, 2022

(CC BY 4.0) | ISSN 2525-3409 | DOI: http://dx.doi.org/10.33448/rsd-v11i2.25185

Tabela 4. Caso 2 - Resultados de Krigagem Ordinária para a Bacia do Rio Paraguai.

\begin{tabular}{cccccc}
\hline PONTO & Estações ID ANA & $\begin{array}{c}\text { Q95 } \\
\left(\mathbf{L} / \mathbf{s . k m}^{2}\right)\end{array}$ & $\begin{array}{c}\mathbf{Q 9 5}^{*} \\
\left(\mathbf{L} / \mathbf{s . k m}^{2}\right)\end{array}$ & $\begin{array}{c}\text { Desvio de } \\
\text { Interpolação }\end{array}$ & $\begin{array}{c}\text { Desvio de } \\
\text { Krigagem }\end{array}$ \\
\hline 1 & 66855000 & 7,73 & 4,94 & 1,23 & 0,83 \\
2 & 66945000 & 2,11 & 2,46 & 1,35 & 1,15 \\
3 & 66840000 & 7,24 & 3,55 & 1,39 & 1,05 \\
4 & 67175000 & 0,27 & 0,43 & 0,39 & 0,87 \\
\hline
\end{tabular}

Fonte: Autores.

Tabela 5. Caso 3 - Resultados de Krigagem Ordinária para Bacia do Rio Paraná.

\begin{tabular}{cccccc}
\hline PONTO & Estações ID ANA & $\begin{array}{c}\text { Q95 } \\
\left(\mathbf{L} / \mathbf{s . k m}^{2}\right)\end{array}$ & $\begin{array}{c}\mathbf{Q 9 5}^{*} \\
\left(\mathbf{L} / \mathbf{s . k m}^{2}\right)\end{array}$ & $\begin{array}{c}\text { Desvio de } \\
\text { Interpolação }\end{array}$ & $\begin{array}{c}\text { Desvio de } \\
\text { Krigagem }\end{array}$ \\
\hline 5 & 64703000 & 9,23 & 8,62 & 0,82 & 1,89 \\
6 & 63950250 & 10,41 & 8 & 0,69 & 2,21 \\
7 & 64610000 & 7,48 & 7,57 & 2,09 & 2,25 \\
8 & 63390000 & 7,39 & 9,99 & 2,34 & 2,42 \\
\hline
\end{tabular}

Fonte: Autores.

Ressalta-se que a baixa disponibilidade de dados para a modelagem pode influenciar negativamente no modelo, acarretando assim uma baixa eficiência, pois os vazios ao longo da malha de pontos amostrais geram regiões com baixa representatividade, dependendo exclusivamente dos parâmetros de vizinhança. As vantagens de se obter os desvios de interpolação através do software possibilitam um melhor ajuste do valor estimado, uma vez que a composição do valor estimado com os desvios traz uma redução significativa do erro relativo.

\section{Conclusão}

A carência por dados hidrológicos se faz cada vez mais presente nos processos de gestão e planejamento de usos de recursos hídricos. A baixa densidade de informações de vazões disponíveis para uso faz com que seja necessário o desenvolvimento de técnicas que permitam a predição de dados em locais com pouca ou nenhuma informação. Baseado nesta necessidade, esta pesquisa propôs a utilização da geoestatística como forma de predição de dados de vazões mínimas em locais sem dados, partindo da premissa de que vazões são variáveis regionalizadas e possuem interdependência devido à localização geográfica.

Aplicando a krigagem ordinária para o mapeamento das vazões é possível ganhar agilidade no processo de obtenção de dados de vazões uma vez que não há a necessidade da obtenção de outros dados hidrológicos para aplicação das metodologias tradicionais de regressão, que necessitam da etapa de definições de regiões homogêneas hidrologicamente.

Para otimizar os modelos, além do mapa obtido para o Estado de MS, foram gerados mapas para as regiões da Bacia do Rio Paraguai e Bacia do Rio Paraná. Através da validação cruzada, foram obtidos índices de correlação em torno de 0,70 indicando um bom ajuste, e que o modelo funciona satisfatoriamente para predição de dados de vazão. A baixa disponibilidade de dados e sua distribuição irregular no espaço foram um agravante para a modelagem dos semivariogramas, acarretando maiores imprecisões em algumas regiões, e por vezes, mesmo com um melhor ajuste do semivariograma foram encontradas poucas diferenças na validação cruzada. A quantidade de dados disponíveis acaba sendo um fator mais determinante na precisão dos resultados do que o próprio modelo de semivariograma escolhido. 
As diferenças encontradas entre os resultados obtidos para a Bacia do Rio Paraguai e a Bacia do Rio Paraná é um fator que explica a complexidade da gestão de recursos hídricos no Estado de Mato Grosso do Sul, uma vez que existem regiões com comportamento hidrológico e disponibilidade de água para uso distintos, havendo assim a necessidade da flexibilização dos critérios de uso e maior cuidado no estudo da transferência de informações de dados hidrológicos de uma região para a outra e elaboração de modelos hidrológicos.

A obtenção dos desvios de interpolação além dos valores estimados para os locais sem dados, possibilitam um intervalo de confiança maior para o trabalho com os dados, possibilitando a incorporação dos desvios aos valores estimados, dando uma maior margem de trabalho.

Futuramente, podem ser gerados novos modelos, utilizando outras formas de krigagem, através de transformações dos dados, procurando sempre otimizar o resultado obtido. Mas, vale ressaltar mais uma vez, a importância do investimento no aumento da malha de dados fluviométricos, fator determinante para a obtenção de resultados mais fidedignos. Além disso, podem ser incorporados aos valores krigados índices que relacionam os valores de vazão com a hidrogeologia local, que influencia diretamente nos valores dessas vazões mínimas.

Neste trabalho é apresentada uma técnica inédita com geoestatística que vem sendo aplicada com sucesso pelo Instituto de Meio Ambiente de Mato Grosso do Sul - IMASUL. O Diagnóstico da Microbacia Hidrográfica do Córrego Água Boa e seus afluentes, trabalho coordenado pelo IMASUL, utilizou a metodologia apresentada neste artigo (IMASUL, 2021).

O Brasil possui locais com climas completamente antagônicos, com regimes de chuvas e disponibilidade hídrica distintas. Dessa forma, é necessário que novas pesquisas sobre predição de vazões sejam elaboradas, para poderem ser aplicadas em programas de governança hídrica, onde são realizados os planejamentos de uso dos recursos hídricos. As técnicas computacionais envolvendo estatística e modelagem de dados hidrológicos são importantes ferramentas que devem ser aplicadas. Todavia, para que os resultados tenham uma maior acurácia é necessário que governos municipais, estaduais e a própria União invistam em trabalhos técnicos para a elaboração e disponibilização e dados hidrológicos, principalmente, para as regiões onde há baixa densidade desse tipo de informação., inclusive em áreas onde não existam esses dados.

\section{Agradecimentos}

Os autores agradecem ao PGTA/UFMS (Programa de Pós-Graduação em Tecnologias Ambientais), da FAGENG, UFMS.

O presente trabalho foi realizado com apoio da Coordenação de Aperfeiçoamento de Pessoal de Nível Superior - Brasil (CAPES) - Código de Financiamento 001 pela bolsa de doutorado de Camila Dourado Machado.

Agradecemos ainda a CAPES pelo acesso ao Portal de Periódicos.

Ao CNPq (Conselho Nacional de Desenvolvimento Científico e Tecnológico) pela bolsa de produtividade em pesquisa de A. C. Paranhos Filho (CNPq Processo 305013/2018-1).

O presente trabalho foi realizado com apoio da Fundação Universidade Federal de Mato Grosso do Sul - UFMS/MEC - Brasil.

\section{Referências}

ANA. (2016). Sistema de Informações Hidrológicas. Agência Nacional das Águas. http://hidroweb.ana.gov.br/default.asp.

Brasil. (1997). Lei $n^{\circ}$ 9.433, de 8 de janeiro de 1997. Institui a Política Nacional de Recursos Hídricos, cria o Sistema Nacional de Gerenciamento de Recursos Hídricos. http://www.planalto.gov.br/ccivil_03/leis/19433.htm.

Brasil. (2003). Conselho Nacional de Recursos Hídricos. RESOLUÇÃO Nº 32, de 15 de outubro de 2003. Diário Oficial da União. 
Research, Society and Development, v. 11, n. 2, e54511225185, 2022

(CC BY 4.0) | ISSN 2525-3409 | DOI: http://dx.doi.org/10.33448/rsd-v11i2.25185

Chaves, H. M. L., Rosa, J. W. C., Vadas, R. G. \& Oliveira, R. V. T. (2002). Regionalização de Vazões Mínimas em Bacias Através de Interpolação em Sistemas de Informação Geográfica. Revista Brasileira de Recursos Hídricos. 7(3),43-51.

Farmer, W. H. (2016). Ordinary kriging as a tool to estimate historical daily streamflow records. Hydrology Earth Syst. Sci. Disponível. https://www.hydrolearth-syst-sci.net/20/2721/2016/.

IBGE (2011) Censo 2010. Instituto Brasileiro de Geografia e Estatística. http://ibge.gov.br/estadosat/perfil.php?sigla=ms.

IMASUL (2014). Resolução CERH/MS N 025, de 20 de outubro de 2014. Diário Oficial [da] República Federativa do Brasil. Instituto de Meio Ambiente de Mato Grosso do Sul Poder Executivo, Brasília, DF, 22 out. 2014, n. 8.784, p. 51- 52. http://www.imasul.ms.gov.br/controle/ShowFile.php?id=175045.

IMASUL (2021). Estudos para subsidiar o enquadramento da microbacia hidrográfica do Córrego Água Boa até a confluência com o Rio Dourados. Instituto de Meio Ambiente do Mato Grosso Do Sul. https://www.imasul.ms.gov.br/wp-content/uploads/2017/03/Diagn\%C3\%B3stico_completo_mbh_agua_boa.pdf.

Qgis Development Team, (2015). QGIS Geographic Information System. Open Source Geospatial Foundation Project. Disponível em < http://www.qgis.org/>. Acesso em 06/12/2021.

Pereira, A. S., Shitsuka, D. M., Parreira, F. J. \& Shitsuka R. (2018). Metodologia da pesquisa científica. UFSM. https://repositorio.ufsm.br/bitstream/handle/1/15824/Lic_Computacao_Metodologia-Pesquisa-Cientifica.pdf?sequence=1.

Silveira, A. L. L. \& Silveira, G. L. (2003). Vazões Mínimas. In: Paiva, J. B. D.; Paiva, E. M. C. D. (Org.) Hidrologia aplicada à gestão de pequenas bacias hidrográficas. Reimpressão revisada. Porto Alegre: ABRH. p. 125-162.

Silveira, G. L., Tucci, C. E. M. \& Silveira, A. L. L. (1998). Quantificação de vazão em pequenas bacias sem dados. Revista Brasileira de Recursos Hídricos. 3 (3): 97-110.

Tucci, C. E. M. (2002). Regionalização de Vazões. Editora UFRGS.

Yamamoto, J. K. (2015). Sistema Geovisual. Versão 5.0. 2015.

Yamamoto, J. K. (2020). Estatística, Análise e Interpolação de Dados Geoespaciais: 50 Scripts em R. São Paulo: Oficina de Textos. 